\title{
Improvement in Bronchiectasis on CT Imaging in a Pediatric Patient with Cystic Fibrosis on Ivacaftor Therapy
}

\author{
Don Hayes Jr. ${ }^{\text {a, b, d }}$ Frederick R. Long ${ }^{c, e} \quad$ Karen S. McCoy ${ }^{a, d} \quad$ Shahid I. Sheikh ${ }^{a, d}$ \\ Departments of ${ }^{a}$ Pediatrics, ${ }^{b}$ Internal Medicine and ${ }^{c}$ Radiology, The Ohio State University College of Medicine, and \\ ${ }^{\mathrm{d}}$ Sections of Pulmonary Medicine, and ${ }^{\text {e}}$ Department of Radiology, Nationwide Children's Hospital, Columbus, Ohio, USA
}

\section{Key Words}

Bronchiectasis - Chest - Children · Computed tomography · Cystic fibrosis $\cdot$ Ivacaftor $\cdot$ Pediatrics

The recent development of ivacaftor therapy, which corrects the gating defect of the cystic fibrosis (CF) transmembrane conductance regulator (CFTR) channel associated with the G551D mutation, has significantly altered the clinical course of CF patients with that specific gene mutation. We present a pediatric patient with $\mathrm{CF}$ who had significant improvement in cylindrical bronchiectasis on CT imaging of the chest.

A 12-year-old female with $\Delta$ F508/G551D genotype was started on ivacaftor treatment with subsequent decrease in sweat chloride 6 months later to 31 from 101 $\mathrm{mmol} / \mathrm{l}$, which had been measured at the time of diagno- sis 11 years earlier. Her body mass index increased from 16.2 to 21.2 after 2 years of therapy, while her spirometry showed improvement in forced vital capacity from 2.02 liters (95\% predicted) to 3.89 liters ( $118 \%$ predicted) and forced expiratory volume in $1 \mathrm{~s}$ from 1.57 liters (82\% predicted) to 3.06 liters (105\% predicted). Respiratory cultures continued to isolate methicillin-resistant Staphylococcus aureus. The year prior to starting ivacaftor therapy, the patient experienced two pulmonary exacerbations requiring oral antibiotics as an outpatient with no further antibiotic therapy required on ivacaftor therapy.

Moreover, CT imaging of the chest (fig. 1) was also a useful tool to appraise response to ivacaftor. We believe the improvement in the lung disease radiographically was possible because the patient had cylindrical bronchiectasis (considered to be early-stage disease) before the development of saccular or varicose bronchiectasis, which is believed to be irreversible.
Fig. 1. CT of the chest without contrast 16 months before (a) and 13 months after (b) starting ivacaftor therapy. Arrows in both panels illustrate the improvement in areas of bronchiectasis in both lungs.
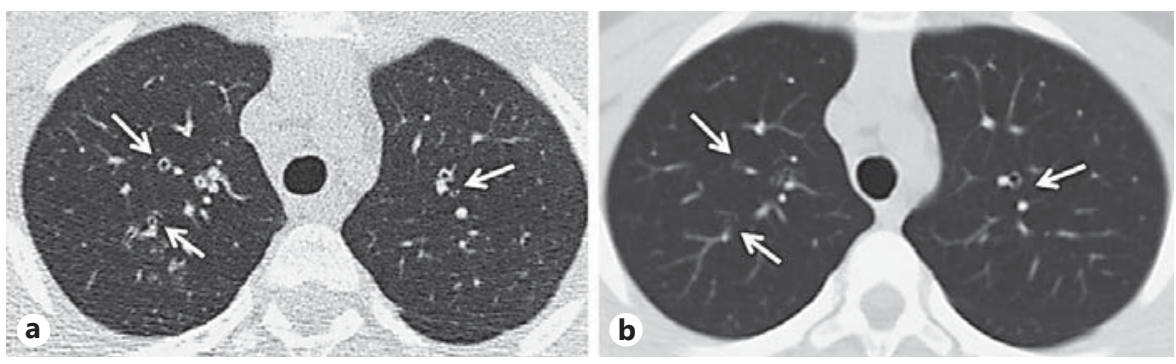

\section{KARGER}

E-Mail karger@karger.com www.karger.com/res
(C) 2014 S. Karger AG, Basel

0025-7931/14/0884-0345\$39.50/0
Don Hayes Jr., MD, MS

Nationwide Children's Hospital

The Ohio State University

700 Children's Drive, Columbus, OH 43205 (USA)

E-Mail hayes.705@osu.edu 